\title{
Kinesin family member 14 induces tumor cell proliferation in muscle invasive bladder cancer
}

\author{
Hai-Tao Zhang, Zhao-Fei Liu, Heng Wang, Si-Nan Hou, Peng-Fei Mao, Rui-Zhi Nie, Xiang-Jun Xu
}

Department of Urology, Lianyungang TCM Hospital Affiliated to Nanjing University of Chinese Medicine, Lianyungang, Jiangsu Province, China

Submitted: 29 January 2020; Accepted: 22 April 2020

Online publication: 10 April 2021

Arch Med Sci

DOI: https://doi.org/10.5114/aoms/120946

Copyright @ 2021 Termedia \& Banach

\section{Abstract}

Introduction: Bladder cancer ranks first in the morbidity of urogenital malignancies in China. Bladder cancers are pathologically classified into 2 subtypes: non-muscle invasion bladder cancer (NMIBC) and muscle invasive bladder cancer (MIBC). MIBC is a highly lethal tumor and targeted therapies are a promising prospect for the treatment of MIBC. Novel therapeutic targets are still badly needed to combat this disease. Kinesin family member 14 (KIF14) is an engaging molecular motor and is involved in multiple cellular processes such as cell division. Additionally, KIF14 is highly expressed in multiple tumor tissues and participates in the progression of several cancers such as gastric cancer and hepatocellular carcinoma. However, its possible role in the development of bladder cancer remains unclear.

Material and methods: Herein, 107 cases of MIBC tissue specimens were collected and detected by immunohistochemistry assays, and we analyzed the relationship between AKIF14 expression and clinical features.

Results: We detected high expression of KIF14 in tumor tissues from patients who underwent MIBC. Furthermore, KIF14 was significantly correlated with clinical features, such as tumor stage $(p=0.001)$. Then we used the cell line T24 and 5637 of bladder cancer in the experimental group transfected with the shKIF14 plasmid. Our results further confirmed the impairment of proliferation capacity after KIF14 ablation in vitro. KIF14, additionally, promoted tumor growth of MIBC in mice.

Conclusions: We surmised that KIF14 could serve as a promising therapeutic target for the treatment of MIBC.

Key words: muscle invasive bladder cancer (MIBC), kinesin family member 14 (KIF14), proliferation, clinical features, therapeutic target.

\section{Introduction}

Bladder cancer ranks first in the morbidity of urogenital malignancies in China [1, 2]. Bladder cancer is biologically and clinically heterogeneous, and causes approximately 330,000 new cases annually and 130,000 deaths annually worldwide $[3,4]$. Bladder cancers are classified into two subtypes according to their pathological features: about $80 \%$ of bladder cancers are non-muscle invasion bladder cancer (NMIBC), whereas the remaining $20 \%$ are muscle invasive bladder cancer (MIBC) $[5,6]$. Nowadays, MIBC is still a highly lethal tumor with high recurrence rates [7]. In fact, despite improvement in therapeutic methods such as surgical techniques, the 5-year overall survival rate of MIBC is low [8, 9]. Recently, targeted therapies showed great promise for the treatment of MIBC [10].

\author{
Corresponding author: \\ Xiang-Jun Xu \\ Department of Urology \\ Lianyungang TCM Hospital \\ Affiliated to Nanjing University \\ of Chinese Medicine \\ No. 160 Chaoyang Middle Road \\ Haizhou District, Lianyungang \\ 222000 Jiangsu Province, China \\ E-mail: xxjnju@qq.com
}


A variety of different targeted drugs have shown good therapeutic effects $[11,12]$. However, given the high heterogeneity and high recurrence rate of MIBC, novel and effective therapeutic targets are still badly needed.

Kinesin proteins are known to be critical for multiple cellular processes such as cargo transport, microtubule dynamics and mitosis [13, 14]. Kinesin family member 14 (KIF14), as a member of this family and an engaging molecular motor, plays key roles in chromosomal segregation, alignment and cytokinesis during cell division $[15,16]$. Interestingly, a previous study indicated that KIF14 could tightly bind to microtubules so as to promote cell division [17]. Additionally, KIF14 mutations could lead to primary microcephaly, severe microcephaly and kidney development defects, suggesting its important function in development [18]. In addition to its effect on development, the potential role of KIF14 in cancer progression has been widely reported [19-26].

Previous studies indicated that KIF14 has an oncogenic role in multiple tumors [19]. KIF14 is highly expressed in tumor tissues of multiple types of cancer, such as prostate cancer, medulloblastoma, and ovarian cancer [20-22]. KIF14 could promote cell proliferation and invasion of several types of cancers including gastric cancer, lung adenocarcinoma, and hepatocellular carcinoma [2325]. Previous studies also indicated the oncogene effect of KIF14 in breast cancer through negatively regulating Rap1a-Radil signaling [26]. Although a large number of studies have reported the roles of KIF14 in tumorigenesis and development, its possible role in the progression and metastasis of bladder cancer remains poorly defined.

Here, we assessed the potential role of KIF14 in the progression of MIBC. We observed that KIF14 was highly expressed in human MIBC tissues and detected a potential link between KIF14 expression and tumor stage of patients with MIBC. We also found that depletion of KIF14 markedly blocked MIBC cell proliferation and suppressed tumor growth in mice. According to our results, a novel and promising therapeutic target, KIF14, was identified for the treatment of MIBC.

\section{Material and methods}

\section{Ethics statement}

All methods conducted in the present study and all the experimental protocols were approved by the Research Ethics Committee of Lianyungang TCM Hospital Affiliated to Nanjing University of Chinese Medicine (Jiangsu Province, China).

\section{Antibodies, primers and shRNA plasmids}

Rabbit anti-KIF14 (for IHC assays, 1:200 dilution, for Immunoblot assays, 1:2000 dilution, \#ab71155, Abcam, Cambridge, UK), rabbit antiKi67 (for IHC assays, 1:100 dilution, for Immunoblot assays, 1:1000 dilution, \#ab16667, Abcam, Cambridge, UK), rabbit anti-proliferating cell nuclear antigen (PCNA) (1:500 dilution, \#ab92552, Abcam, Cambridge, UK), and mouse anti- $\beta$-actin (1:1000 dilution, \#ab8226, Abcam, Cambridge, UK) were used.

The quantitative PCR primer sequences targeting KIF14 were as follows: forward, 5'-CCGACATTACAGATGCACCA-3' and reverse, 5'-CTTCATTCCTAAGCCTACACC-3'; The quantitative PCR primer sequences of GAPDH were as follows: $5^{\prime}$-TGCACCACCACCTGCTTAGC-3' and 5'-GGCATGGACTGTGGTCATGAG-3'.

Ready-to-package AAV shRNA plasmids targeting KIF14 were purchased from Addgene plc. The shRNA sequence targeting KIF14 was as follows: sense, 5'-TTCCTTCTTCCCAAAATAGTTCA -3'.

\section{Human tissue samples and analysis}

The 107 human MIBC and corresponding non-tumor tissue samples studied in this study were collected from the patients receiving surgical treatment in our hospital. The clinical-pathological characteristics, such as patient age, gender, and tumor stage, are listed in Table I.

To explore the possible correlations between the expression levels of KIF14 and MIBC progression, immunohistochemical (IHC) assays were performed. Briefly, sample sections were fixed with 4\% PFA for 30 min and subsequently blocked with $2 \%$ BSA for $30 \mathrm{~min}$. Slides were then incubated with KIF14 and Ki67 antibodies at room temperature for $2 \mathrm{~h}$. Subsequently the sections were incubated with biotinylated secondary antibody for $1.5 \mathrm{~h}$, and diaminobenzidine was used as a chromogen substrate.

Kinesin family member 14 was found located in both the cytoplasm and nucleus of MIBC tissues. The scoring methods were as follows. In brief, the percentage of positively stained cells was graded as follows: $0-0 \%$ stained cells; $1-1-30 \%$ stained cells; 2 - 31-60\% stained cells; 3 - 61-100\% stained cells. The staining intensity was further evaluated on a score of 0 - no staining, 1 - low level staining, 2 - moderate level staining, and 3 high level staining. The expression levels of KIF14 were examined based on the staining index: score of staining intensity + score of stained cells percentage. Staining index $<3$ was considered relatively low expression, while staining index 3 or $>3$ was considered high expression.

\section{Cell culture and transfection}

The human MIBC cell lines, T24 and 5637, were bought from ATCC. T24 and 5637 cells were main- 
Table I. Relationships of KIF14 and clinicopathological characteristics in 107 patients with muscular invasive bladder cancer

\begin{tabular}{|c|c|c|c|c|c|}
\hline \multirow[t]{2}{*}{ Feature } & \multirow{2}{*}{$\begin{array}{c}\text { All } \\
N=107\end{array}$} & \multicolumn{2}{|c|}{ KIF14 expression } & \multirow[t]{2}{*}{$\chi^{2}$} & \multirow[t]{2}{*}{$P$-value } \\
\hline & & $\begin{array}{c}\text { Low } \\
n=32\end{array}$ & $\begin{array}{c}\text { High } \\
n=75\end{array}$ & & \\
\hline Age [years]: & & & & 2.212 & 0.137 \\
\hline$<65$ & 42 & 16 & 26 & & \\
\hline$\geq 65$ & 65 & 16 & 49 & & \\
\hline Gender: & & & & 0.001 & 0.981 \\
\hline Male & 60 & 18 & 42 & & \\
\hline Female & 47 & 14 & 33 & & \\
\hline Tumor stage: & & & & 6.639 & $0.010^{*}$ \\
\hline $\mathrm{T} 2$ & 47 & 8 & 39 & & \\
\hline $\mathrm{T} 3 / \mathrm{T} 4$ & 60 & 24 & 36 & & \\
\hline Tumor grade: & & & & 1.222 & 0.269 \\
\hline Low & 29 & 11 & 18 & & \\
\hline High & 78 & 21 & 57 & & \\
\hline $\begin{array}{l}\text { Lymph node } \\
\text { Metastasis: }\end{array}$ & & & & 2.318 & 0.128 \\
\hline Yes & 31 & 6 & 25 & & \\
\hline No & 76 & 26 & 50 & & \\
\hline Recurrence: & & & & 2.123 & 0.145 \\
\hline Yes & 55 & 13 & 42 & & \\
\hline No & 52 & 19 & 33 & & \\
\hline
\end{tabular}

tained in Dulbecco's modified essential medium (DMEM) and RPMI-1640 medium, respectively, supplemented with $10 \%$ fetal bovine serum (FBS, Gibco, CA, USA) in a $5 \% \mathrm{CO}_{2}$ incubator.

The KIF14 shRNA plasmids were transfected into both T24 and 5637 cells using Lipofectamine 2000 (\#11668019, Invitrogen, CA, USA). KIF14 stably depleted T24 cells were screened for shRNA lentivirus infection and used for the tumor growth assays in vivo.

\section{Quantitative PCR assay}

Trizol (\#15596026, Invitrogen, CA, USA) was used to extract total RNA from human MIBC cells. Subsequently the RNA was reverse-transcribed by M-MLV reverse transcriptase (\#M1701, Promega, Wisconsin, USA).

Total mRNA was reverse transcribed to produce cDNA by a cDNA synthesis system. Quantitative PCR was performed through a SYBR Ex Taq kit (\#638319, Takara, Japan), and the KIF14 expression level was normalized to the expression of GAPDH.

\section{Immunoblot assays}

Tumor cells or tissues of MIBC were lysed in RIPA Buffer (\#9800, Cell Signaling, Danvers, MA). Then the total proteins were analyzed by SDS-PAGE as- says. Subsequently the polyvinylidene fluoride (PVDF) membranes were blocked with 5\% milk buffer and then incubated with the primary antibodies for the detection of KIF14, Ki67, PCNA, and $\beta$-actin at room temperature for $2 \mathrm{~h}$. Then the PVDF membranes were incubated with HRP-conjugated secondary antibodies for $1 \mathrm{~h}$. Blots were detected with an ECL kit. Image Pro software was used in this assay to calculate the intensity of each blot.

\section{Colony formation assay}

Approximately 1000 cells were added to a 6-well culture plate and transfected with control or KIF14 shRNA plasmids and cultured at $37^{\circ} \mathrm{C}$. The medium was refreshed with fresh medium every 2 days. After 2 weeks, cells were fixed with PFA for $30 \mathrm{~min}$ and stained with $0.2 \%$ crystal violet at room temperature for $20 \mathrm{~min}$ and washed with PBS twice. Then the number of colonies was manually counted and analyzed.

\section{MTT assay}

T24 and 5637 cells were plated into 96-well plates with a density of about 500 cells per well, transfected with control or KIF14 shRNA plasmids and maintained for $48 \mathrm{~h}$. Cells were then incubated with MTT for $4 \mathrm{~h}$ and the medium removed. Subsequently, cells were washed with PBS. Then 
$100 \mu \mathrm{l}$ of dimethyl sulfoxide (DMSO) was added into each well to extract the stained cells, and the absorbance value was measured with a microplate reader at $570 \mathrm{~nm}$ wavelength.

\section{Tumor growth assays}

All animal assay processes were approved by our Institutional Animal Care and Use Committee (IACUC). Briefly, T24 cells were stably infected with control or KIF14 shRNA lentivirus. Subsequently, about $10^{6} \mathrm{~T} 24$ cells were subcutaneously implanted into athymic nude mice. After 14 days, tumors were isolated, and photographed every 3 days. After 29 days, the growth curves were calculated and compared.

\section{Statistical analysis}

GraphPad 5.0 software was used for statistical analysis in this study. All results were presented as mean \pm standard deviation (SD). The correlations between clinical features and KIF14 expression levels were calculated using $\chi^{2}$ analysis. Student's $t$-test was used for statistical comparisons; ${ }^{*}$ indicates $p<0.05$, which was considered as a statistically significant difference.

\section{Results}

\section{Significant up-regulation of KIF14} expression was found in human MIBC tumor tissues, and KIF14 was correlated with the prognosis of patients with MIBC

To assess the potential role of KIF14 in tumor progression, we first conducted bioinformatic analysis using the TCGA database (http://gepia.cancer-pku.cn/detail.php?gene=KIF14): Bioinformatic analysis provided evidence that KIF23 mRNA level is enhanced in human bladder urothelial carcinoma (BLCA) tissues $(n=404)$ compared to normal tissues $(n=28)$ (Supplementary Figure S1 A, $p<0.05)$, and it is correlated with a poor prognosis: KIF14 is associated with the disease-free survival (DFS) rate in samples with different numbers of cases (Supplementary Figure S1 B, $p=0.019$, $<0.05$, respectively). Then, to assess the potential role of KIF14 in tumor progression in China, KIF14 expression levels in MIBC tissues of patients who underwent surgical resection were examined through IHC assays. We initially found that KIF14 was mainly located in the cytoplasm of MIBC cells (Figure $1 \mathrm{~A}$ ). To further evaluate the effects of KIF14 in MIBC development, we detected the difference of KIF14 expression levels between MIBC tissues and the adjacent tissues through IHC assays. Consistent with our expectations, MIBC tissues showed relatively high expression levels of KIF14 compared with adjacent tissues (Figures $1 \mathrm{~A}, \mathrm{~B}$ ).
According to the staining results, a total of 107 tissue samples taken from MIBC patients who underwent surgical treatment were manually classified into KIF14 low and high expression groups (Figure $1 \mathrm{~A}$ and Table I). Based on the staining intensity of KIF14 in tumor tissues, 32 patients showed low expression of KIF14, whereas 75 exhibited high KIF14 expression levels. We subsequently assessed the clinical significance of KIF14 in MIBC patients. Clinical characteristics including patient age, gender, and tumor size were analyzed. However, no clinical significant difference was found in features such as patient age and gender between these two groups. Interestingly, our results revealed that the expression level of KIF14 was significantly associated with tumor stage $(p=0.01,<0.05)$ in MIBC patients (Table I).

Collectively, the possible significance of the association between KIF14 expression and tumor stage was found in MIBC patients.

\section{KIF14 contributes to tumor cell proliferation of MIBC in vitro}

To further evaluate the mechanism underlying KIF14 promoting the progression of MIBC, the KIF14 shRNA plasmids were transfected into two types of human MIBC cell lines, T24 and 5637, to suppress its expression levels. Through quantitative PCR assays, we found that the transfection of KIF14 shRNA plasmids effectively blocked its expression in both T24 and 5637 cells (Figure 2 A). Similarly, the results of Immunoblot assays further proved the obviously reduced expression levels of KIF14 in both T24 and 5637 cells transfected with its shRNA plasmids (Figure $2 \mathrm{~B}$ ).

Subsequently, colony formation assays were performed to examine the proliferation capacity of MIBC cells. We found that the KIF14 depletion effectively decreased the colony number through colony formation assays (Figure $3 \mathrm{~A}$ ). Similarly, an obvious reduced absorbance value at $570 \mathrm{~nm}$ was detected in both T24 and 5637 cells through MTT assays (Figure $3 \mathrm{~B}$ ). Meanwhile, the expression levels of Ki67 and PCNA, two bio-markers reflecting proliferate capacity, were detected through Immunoblot assays. We found that knockdown of KIF14 resulted in the significant reduction of Ki67 and PCNA expression levels in T24 and 5637 cells, respectively (Figures $3 \mathrm{C}, \mathrm{D}$ ).

In conclusion, we found that KIF14 contributed to cell proliferation of MIBC in vitro.

\section{Depletion of KIF14 inhibited tumor growth of MIBC in mice}

We then explored the possibility that KIF14 promoted tumor growth of MIBC through animal assays. 
A
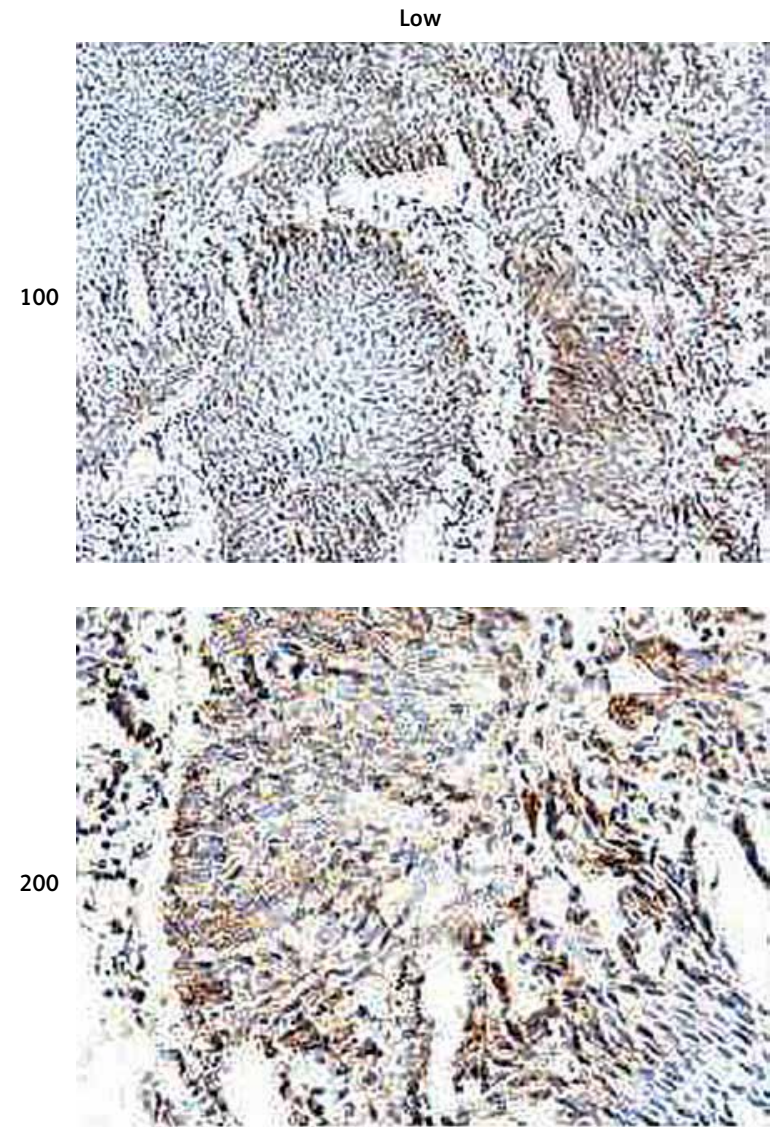

High
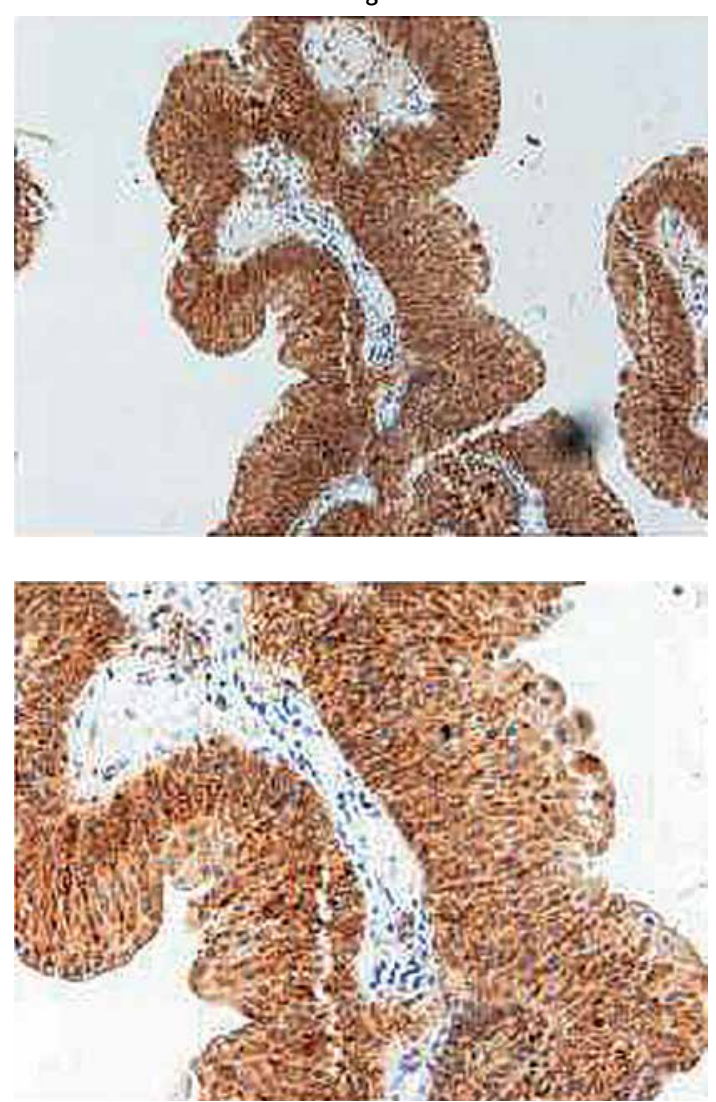

B

100
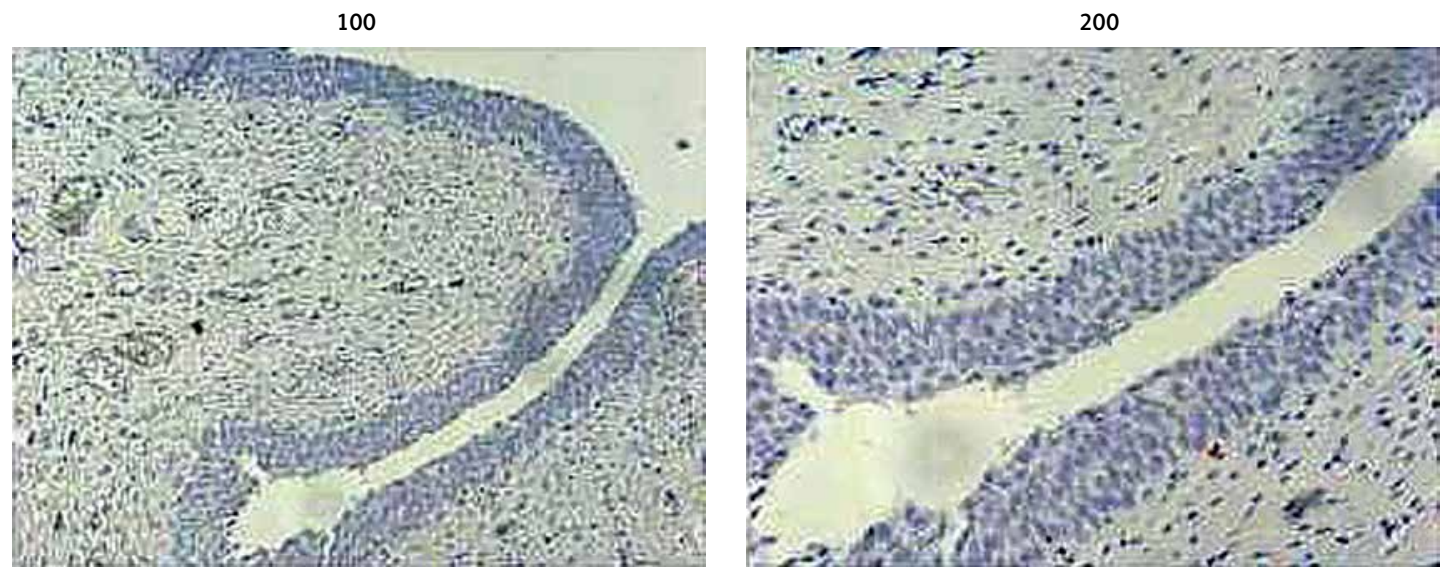

Figure 1. KIF14 was highly expressed in human MIBC tissues. A - Immunohistochemical assays were performed, and representative photographs of KIF14 in human MIBC tissues were taken and shown $(100 \times$ and $200 \times$ magnification). B - Immunohistochemical staining showed the expression levels of KIF14 in non-tumor tissues (100 × and $200 \times$ magnification)

To confirm our hypothesis, T24 cells were infected with KIF14 or control shRNA lentivirus and subcutaneously injected into nude mice. After 2 weeks, the tumor was isolated, photographed, and the volume was measured every 3 days. After 29 days, all tumors are isolated and representative photographs were then shown (Figure $4 \mathrm{~A}$ ). The growth curve was also calculated and shown in Figure $4 \mathrm{~A}$. Interestingly, tumor volume in KIF14 depletion groups was markedly smaller than in the control group (Figure $4 \mathrm{~A}$ ).

Further, IHC assays exhibited the effective silencing of KIF14 in tumor tissues from KIF14 shRNA lentivirus infected groups (Figure 4 B). We measured the expression levels of both Ki67 and PCNA in tumor tissues from control and KIF14 knockdown groups by IHC assays. Interestingly, the significant decrease of Ki67 expression levels 

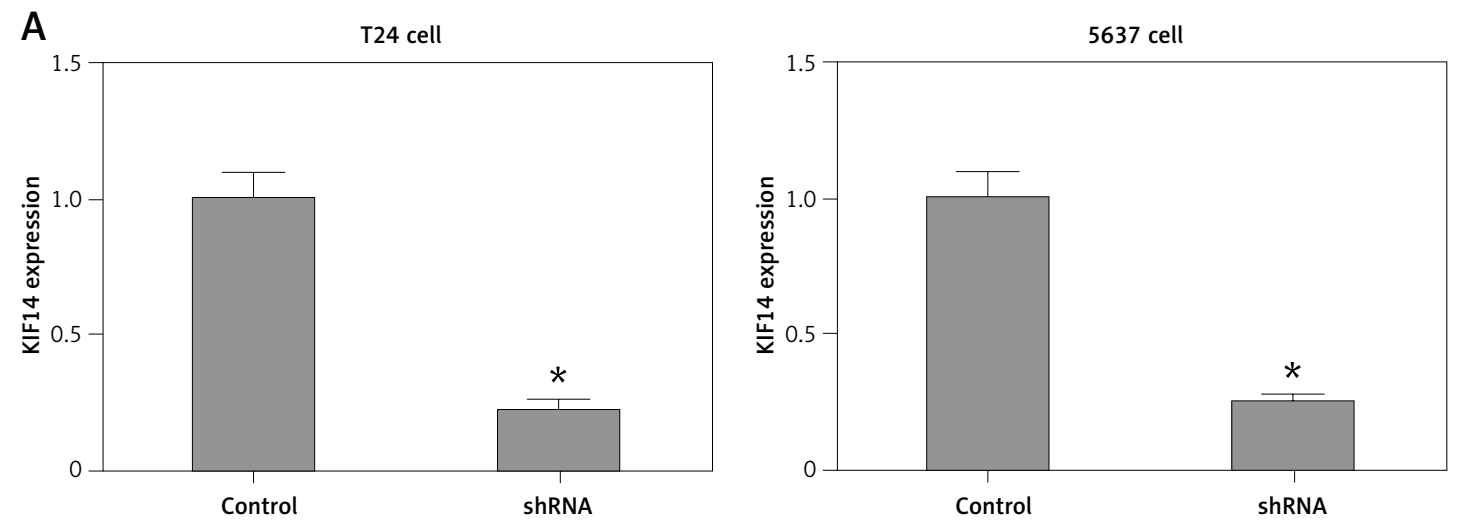

B
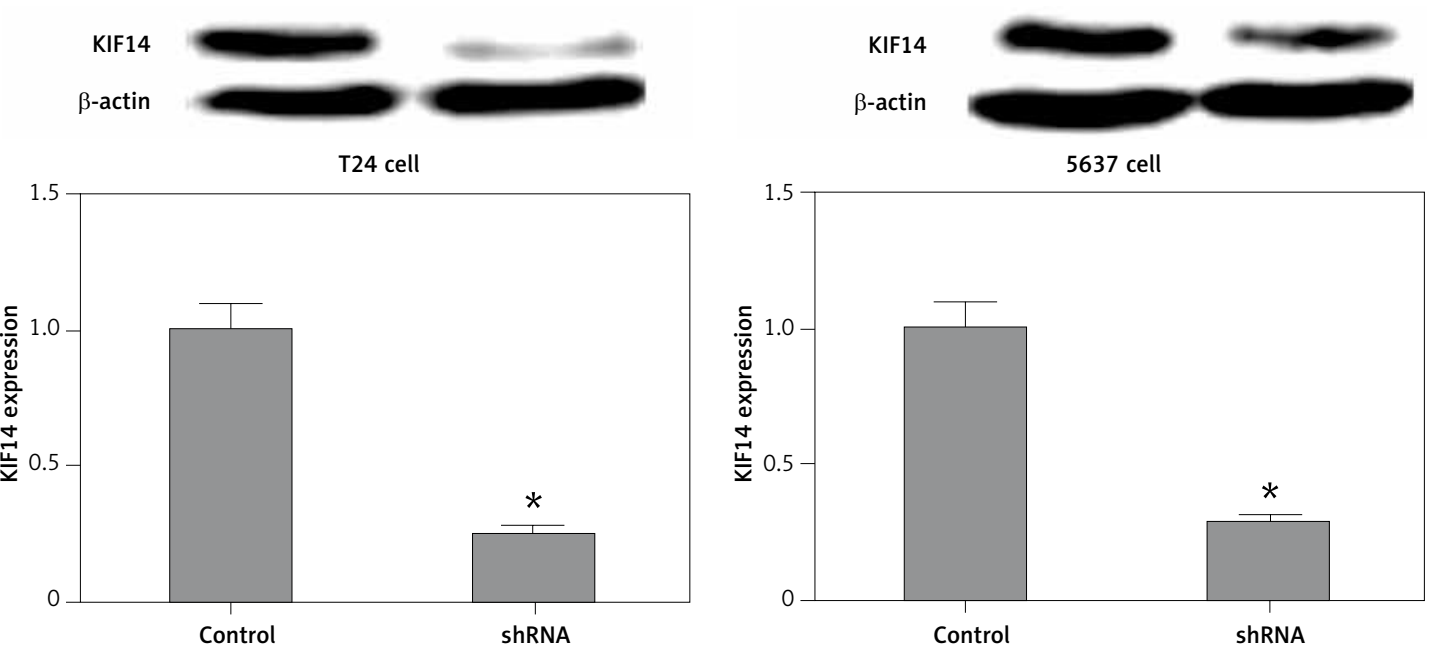

Figure 2. KIF14 expression levels were significantly blocked in both T24 and 5637 human MIBC cells after KIF14 knockdown. A - Quantitative PCR assays revealed the obviously decreased expression levels of KIF14 following the transfection of its shRNA plasmids in T24 and 5637 cells. B - Immunoblot assays showed the efficient inhibition of KIF14 expression after the transfection of KIF14 shRNA plasmids in both T24 and 5637 cells

Results are presented as mean $\pm S D ;{ }^{*} p<0.05$.

A
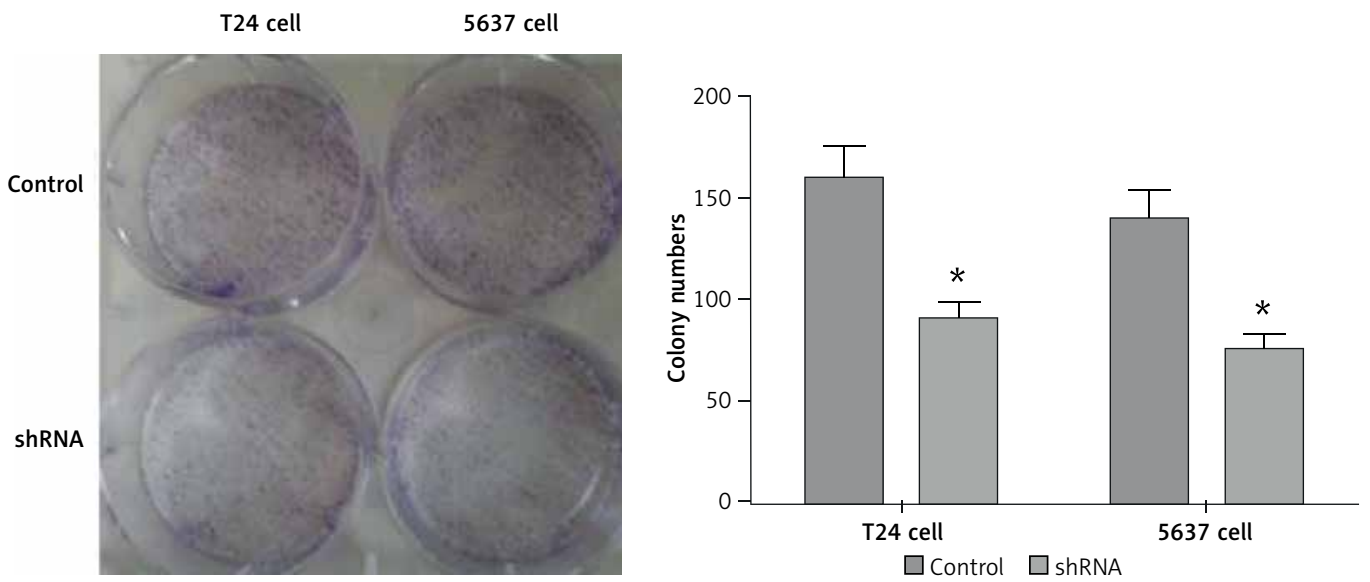

Figure 3. KIF14 promotes cell proliferation of MIBC in vitro. A - T24 and 5637 cells were transfected with control or KIF14 shRNA plasmids, and colony formation assays were then performed

Results are presented as mean $\pm S D ;{ }^{*} p<0.05$. 

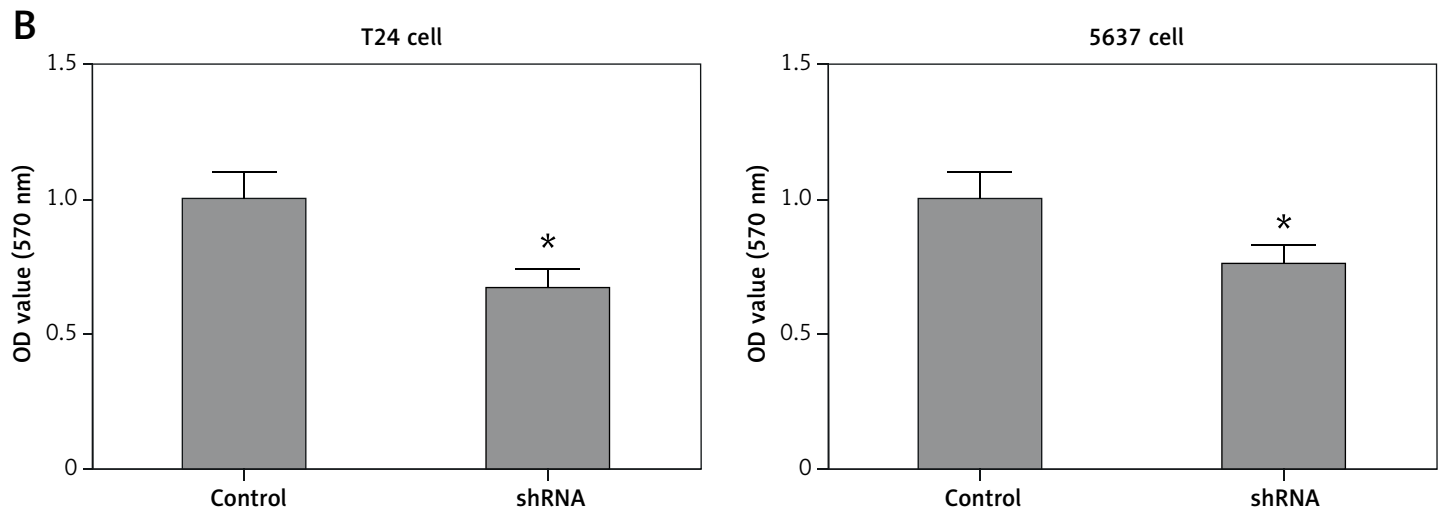

C

Ki67

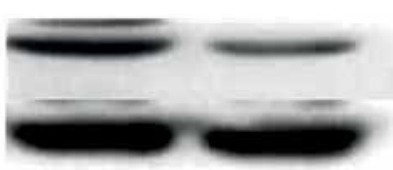

Ki67

$\beta$-actin
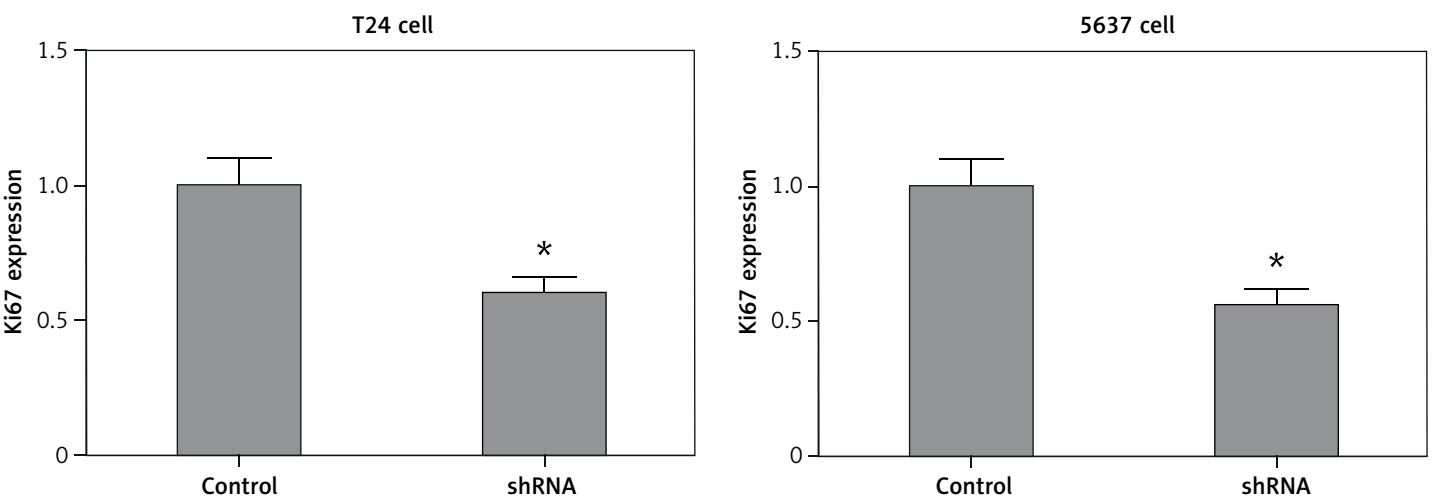

D

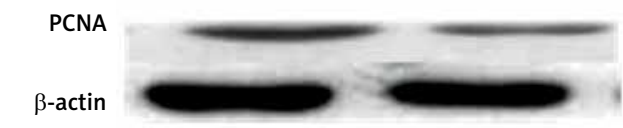

PCNA

$\beta$-actin

T24 cell
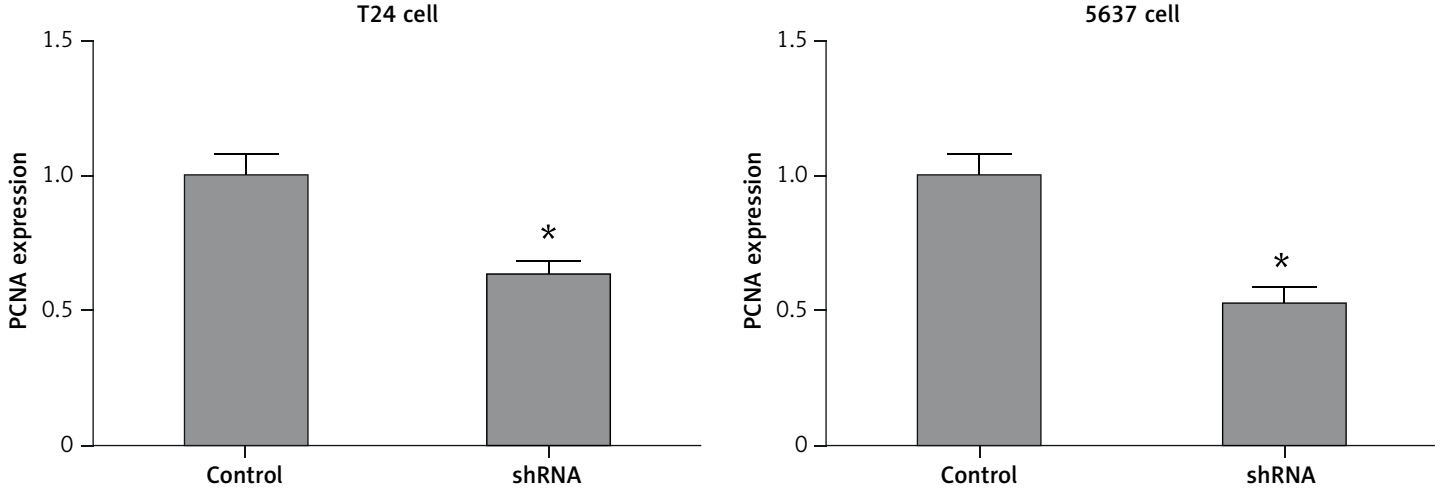

Figure 3. Cont. B - The results of MTT assays exhibited the decreased cell proliferation capacity caused by KIF14 depletion. C, D - Immunoblot assays revealed Ki67 and PCNA expression levels in the indicated shRNA treated T24 and 5637 cells, respectively

Results are presented as mean $\pm S D ;{ }^{*} p<0.05$. 

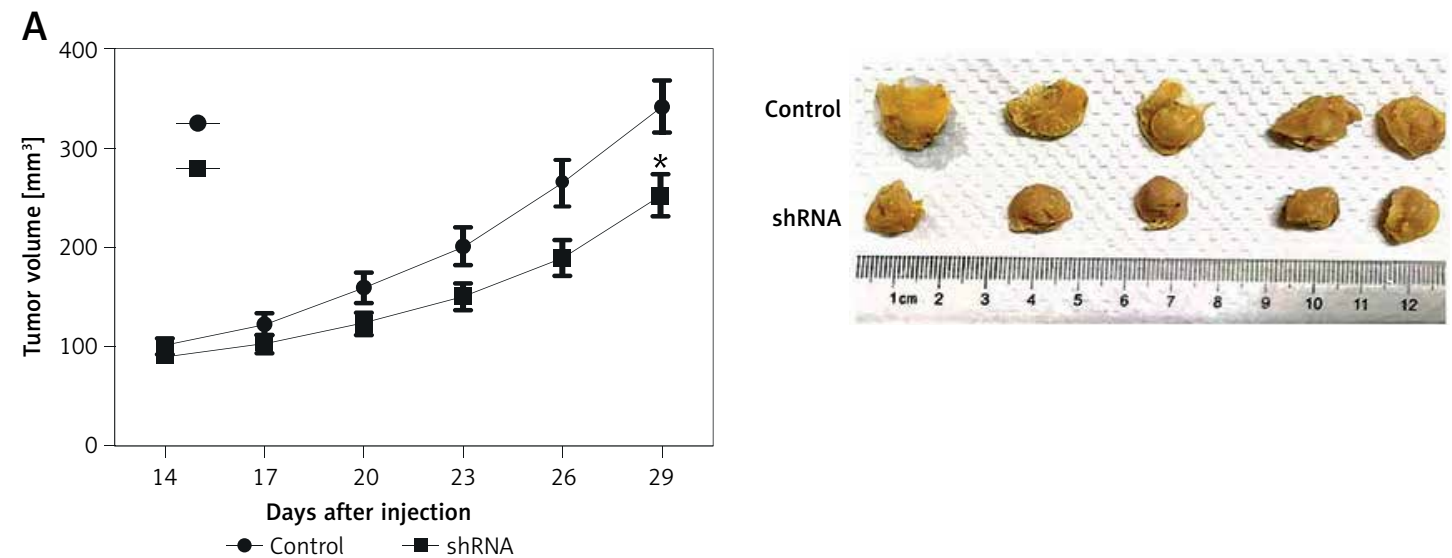

B
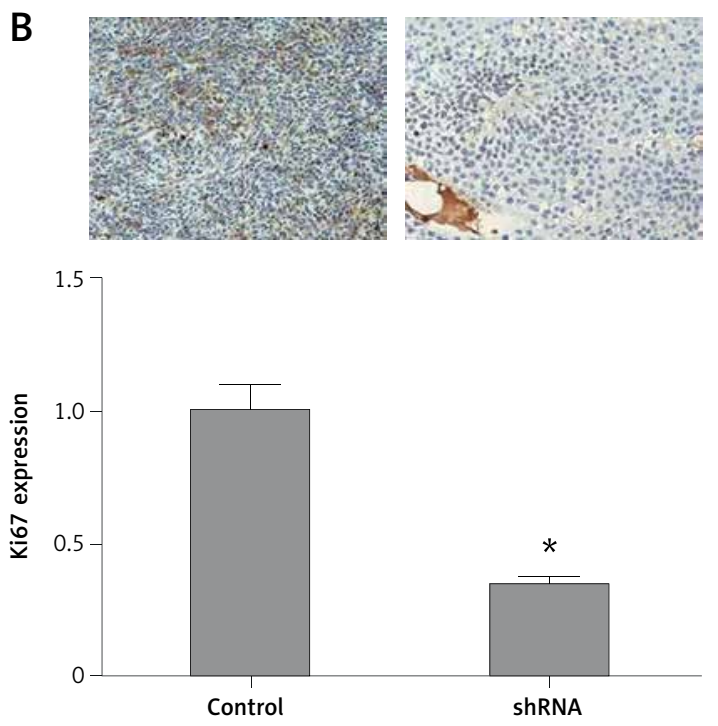

C
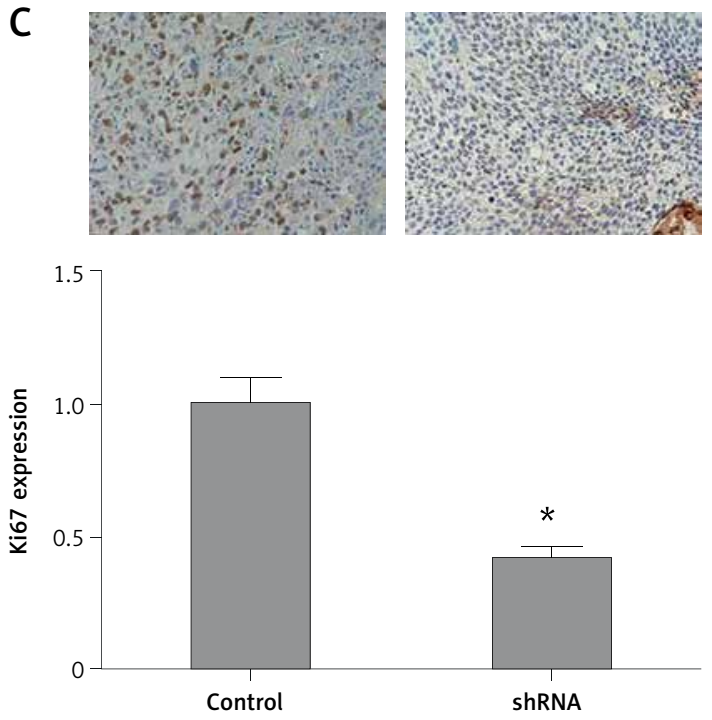

Figure 4. KIF14 induces tumor growth of MIBC in mice. A - T24 cells infected with control or KIF14 shRNA lentivirus were subcutaneously implanted into nude mice. After 2 weeks, tumors were isolated, and the volume of tumor was measured every 3 days ( $n=5$ in each group). After 29 days, tumor growth curves were calculated and analyzed based on the average volume of 5 tumors in KIF14 knockdown and control groups. B - IHC assays revealed the expression levels of KIF14 in control or KIF14 depletion tumors isolated from mice. C - IHC assays showed the expression levels of Ki67 in control or KIF14 ablation tumors isolated from mice

Results are presented as mean $\pm S D ;{ }^{*} p<0.05$.

was detected in tumors from KIF14 knockdown groups, suggesting that the proliferation capacity of tumor cells was weaker after KIF14 knockdown (Figure $4 \mathrm{C}$ ). In summary, the results of our in vivo assays indicated the potential involvement of KIF14 in tumor growth of MIBC in mice.

\section{Discussion}

In this study, we evaluated the possible correlation of KIF14 expression with pathological and clinical features in bladder cancer. We found that KIF14 was not only highly expressed in human tumor tissues of MIBC, but also correlated with tumor stage. We further found here that depletion of KIF14 by its shRNA strongly inhibited proliferation of T24 and 5637 cells through colony formation and MTT assays. The potential role of KIF14 as a proliferation bio-marker is also revealed by the significant relationship found between KIF14, Ki67, and PCNA expression levels. Similarly, we confirmed the anti-proliferation effects of KIF14 shRNA lentivirus in mice. Together, these in vitro and in vivo findings suggested that KIF14 could be a very promising target for MIBC therapeutic development, whereas the precise regulatory mechanism of KIF14 in MIBC progression needs further study.

To our knowledge, this is the first report of a link between KIF14 expression and the progression of bladder cancer. As a molecular motor, KIF14 has been widely reported in the progression and development of multiple cancers [20-26]. KIF14 expression level is a predictor of the prognosis of breast cancer and ovarian cancer $[22,26]$. Another study also indicated that KIF14 contributes to AKT phosphorylation in triple-negative breast cancer 
(TNBC) [23]. KIF14, notably, promotes tumor cell proliferation and invasion of breast cancer through the Rap1a-Radil signaling pathway [26]. We here observed that KIF14 promoted cell proliferation of bladder cancer, and whether through Rap1a-Radil or AKT signaling pathways needs to be studied in future. In addition, previous studies also reported that KIF14 suppressed tumor growth and metastasis in lung cancer through recruiting adhesion molecules to the cell membrane and modulating cell adhesive and invasive properties [24]. Whether KIF14 induces MIBC metastasis through a similar mechanism is worth further exploration.

As we know, KIF14 plays important roles in cell cycle progression and cell division, and the inhibition of KIF14 expression could lead to a number of human diseases including malignancies [25]. KIF14, as a member of the kinesin family, was first cloned in 1994 and is characterized by a C-terminal citron kinase binding domain and $\mathrm{N}$-terminal motor domain; therefore KIF14 has the capacity to bind microtubules and affect cell division [27]. Here we found that KIF14 promoted cell proliferation of bladder cancer, possibly through its role in cell division. Similarly, there was also a study indicating that KIF14 expression obviously varied during the cell cycle, with peak expression in S phase, and its depletion led to distinct phenotypes depending on the degree of ablation of KIF14 [25]. We next should detect the effects of KIF14 depletion on the cell cycle, which will perhaps explain the proliferation defects caused by KIF14 knockdown.

Except for KIF14, multiple members of the kinesin family are involved in tumorigenesis and could serve as prognostic predictors of several types of cancer [28]. High expression of KIF22 and KIF15 could be poor prognostic factors in patients with prostate cancer and lung adenocarcinoma, respectively [29, 30]. In addition, KIF20A is correlated with an unfavorable clinical outcome and progression of epithelial ovarian cancer [31]. KIF26B depletion could suppress cell proliferation, migration and invasion of breast cancer [32]. Recently, we have seen similar published articles [33, 34]. These studies, together with our findings in this study, indicate the key roles of kinesins in tumorigenesis and development, and suggest that the development of inhibitors targeting kinesins could be a promising prospect in future.

Collectively, our data indicated the high expression levels of KIF14 in human MIBC tumor tissues. We investigated the possible link between KIF14 expression levels and clinical characteristics of MIBC patients. Also, KIF14 stimulated cell proliferation of cancer in vitro and promoted tumor growth of MIBC in mice. We therefore provided a mechanical exploration of KIF14 in the progres- sion of MIBC and a novel molecular target for MIBC.

\section{Conflict of interest}

The authors declare no conflict of interest.

\section{References}

1. Li C, Wang Z, Feng N, et al. Human HLAF adjacent transcript 10 promotes the formation of cancer initiating cells and cisplatin resistance in bladder cancer. Mol Med Rep 2018; 18: 308-14.

2. Liu XP, Yin XH, Meng XY, et al. DHCR24 predicts poor clinicopathological features of patients with bladder cancer: a STROBE-compliant study. Medicine (Baltimore) 2018; 97: e11830.

3. Tseng $\mathrm{CH}$. Human insulin does not increase bladder cancer risk. PLoS One 2014; 9: e86517.

4. Chang Y, Xu J, Zhang Q. Microplate magnetic chemiluminescence immunoassay for detecting urinary survivin in bladder cancer. Oncol Lett 2017; 14: 4043-52.

5. Nargund VH, Tanabalan CK, Kabir MN. Management of non-muscle-invasive (superficial) bladder cancer. Semin Oncol 2012; 39: 559-72.

6. Duenas M, Martinez-Fernandez M, Garcia-Escudero R, et al. PIK3CA gene alterations in bladder cancer are frequent and associate with reduced recurrence in non-muscle invasive tumors. Mol Carcinog 2015; 54: 566-76.

7. Tian DW, Liu SL, Jiang LM, et al. RAB38 promotes bladder cancer growth by promoting cell proliferation and motility. World J Urol 2019; 37: 1889-97.

8. Mahran A, Bukavina L, Mishra K, et al. Bladder irrigation after transurethral resection of superficial bladder cancer: a systematic review of the literature. Can J Urol 2018; 25: 9579-84.

9. Bazargani ST, Clifford TG, Djaladat $\mathrm{H}$, et al. Association between precystectomy epithelial tumor marker response to neoadjuvant chemotherapy and oncological outcomes in urothelial bladder cancer. Urol Oncol 2019, 37: 1-11.

10. Rebouissou S, Bernard-Pierrot I, de Reynies A, et al. EGFR as a potential therapeutic target for a subset of muscle-invasive bladder cancers presenting a basal-like phenotype. Sci Transl Med 2014; 6: 244ra291.

11. Kim WT, Seo SP, Byun YJ, et al. The Anticancer effects of garlic extracts on bladder cancer compared to cisplatin: a common mechanism of action via centromere protein M. Am J Chin Med 2018; 46: 689-705.

12. Ineichen GB, Rothlisberger R, Johner KF, Seiler R. Different stages in drug development for muscle-invasive bladder cancer. Transl Androl Urol 2017; 6: 1060-6.

13. Al-Obaidi N, Kastl J, Mayer TU. Small molecule approach to study the function of mitotic kinesins. Methods $\mathrm{Mol}$ Biol 2016; 1413: 283-99.

14. Goshima G. [Roles of kinesins in mitosis]. Tanpakushitsu Kakusan Koso 2006; 51 (6 Suppl.): 579-85.

15. Rice S. Structure of kif14: an engaging molecular motor. J Mol Biol 2014; 426: 2993-6.

16. Miyamoto I, Kasamatsu A, Yamatoji $M$, et al. Kinesin family member 14 in human oral cancer: A potential biomarker for tumoral growth. Biochem Biophys Rep 2015; 3: 26-31.

17. Arora K, Talje L, Asenjo AB, et al. KIF14 binds tightly to microtubules and adopts a rigor-like conformation. J Mol Biol 2014; 426: 2997-3015. 
18. Reilly ML, Stokman MF, Magry V, et al. Loss of function mutations in KIF14 cause severe microcephaly and kidney development defects in humans and zebrafish. Hum Mol Genet 2019; 28: 778-95.

19. Wang W, Shi Y, Li J, Cui W, Yang B. Up-regulation of KIF14 is a predictor of poor survival and a novel prognostic biomarker of chemoresistance to paclitaxel treatment in cervical cancer. Biosci Rep 2016, 36: e00315.

20. Zhang Y, Yuan Y, Liang P, et al. Overexpression of a novel candidate oncogene KIF14 correlates with tumor progression and poor prognosis in prostate cancer. Oncotarget 2017; 8: 45459-69.

21. Li KK, Qi Y, Xia T, et al. The kinesin KIF14 is overexpressed in medulloblastoma and downregulation of KIF14 suppressed tumor proliferation and induced apoptosis. Lab Invest 2017; 97: 946-61.

22. Theriault BL, Basavarajappa HD, Lim H, Pajovic S, Gallie BL, Corson TW. Transcriptional and epigenetic regulation of KIF14 overexpression in ovarian cancer. PLoS One 2014; 9: e91540.

23. Yang Z, Li C, Yan C, et al. KIF14 promotes tumor progression and metastasis and is an independent predictor of poor prognosis in human gastric cancer. Biochim Biophys Acta Mol Basis Dis 2019; 1865: 181-92.

24. Hung PF, Hong TM, Hsu YC, et al. The motor protein KIF14 inhibits tumor growth and cancer metastasis in lung adenocarcinoma. PLoS One 2013; 8: e61664.

25. Xu H, Choe C, Shin SH, et al. Silencing of KIF14 interferes with cell cycle progression and cytokinesis by blocking the p27(Kip1) ubiquitination pathway in hepatocellular carcinoma. Exp Mol Med 2014; 46: e97.

26. Ahmed SM, Theriault BL, Uppalapati M, et al. KIF14 negatively regulates Rap1a-Radil signaling during breast cancer progression. J Cell Biol 2012; 199: 951-67.

27. She ZY, Yang WX. Molecular mechanisms of kinesin-14 motors in spindle assembly and chromosome segregation. J Cell Sci 2017; 130: 2097-110.

28. Rath O, Kozielski F. Kinesins and cancer. Nat Rev Cancer 2012; 12: 527-39.

29. Zhang Z, Xie H, Zhu S, et al. High expression of KIF22/ kinesin-like DNA binding protein (Kid) as a poor prognostic factor in prostate cancer patients. Med Sci Monit 2018; 24: 8190-7.

30. Qiao Y, Chen J, Ma C, et al. Increased KIF15 expression predicts a poor prognosis in patients with lung adenocarcinoma. Cell Physiol Biochem 2018; 51: 1-10.

31. Li H, Zhang W, Sun $X$, et al. Overexpression of kinesin family member 20A is associated with unfavorable clinical outcome and tumor progression in epithelial ovarian cancer. Cancer Manag Res 2018; 10: 3433-50.

32. Gu S, Liang H, Qi D, et al. Knockdown of KIF26B inhibits breast cancer cell proliferation, migration, and invasion. Onco Targets Ther 2018; 11: 3195-203.

33. Xin F, Yao DW, Fan L, Liu JH, Liu XD. Adenylate kinase 4 promotes bladder cancer cell proliferation and invasion. Clin Exp Med 2019; 19: 525-34.

34. Hu H, Meng Q, Lei T, Zhang M. Nucleophosmin1 associated with drug resistance and recurrence of bladder cancer. Clin Exp Med 2015; 15: 361-9. 